University of Nebraska - Lincoln

DigitalCommons@University of Nebraska - Lincoln

$10-2011$

\title{
Electrical control of photoluminescence wavelength from semiconductor quantum dots in a ferroelectric polymer matrix
}

\author{
Rafal Korlacki \\ University of Nebraska-Lincoln, rkorlacki2@unl.edu \\ Ravi F. Saraf \\ University of Nebraska-Lincoln, rsaraf2@unl.edu \\ Stephen Ducharme \\ University of Nebraska-Lincoln, sducharme1@unl.edu
}

Follow this and additional works at: https://digitalcommons.unl.edu/physicsducharme

Part of the Physics Commons

Korlacki, Rafal; Saraf, Ravi F.; and Ducharme, Stephen, "Electrical control of photoluminescence wavelength from semiconductor quantum dots in a ferroelectric polymer matrix" (2011). Stephen Ducharme Publications. 73.

https://digitalcommons.unl.edu/physicsducharme/73

This Article is brought to you for free and open access by the Research Papers in Physics and Astronomy at DigitalCommons@University of Nebraska - Lincoln. It has been accepted for inclusion in Stephen Ducharme Publications by an authorized administrator of DigitalCommons@University of Nebraska - Lincoln. 


\title{
Electrical control of photoluminescence wavelength from semiconductor quantum dots in a ferroelectric polymer matrix
}

\author{
Rafał Korlacki, ${ }^{1,2, a)}$ Ravi F. Saraf, ${ }^{2}$ and Stephen Ducharme ${ }^{1}$ \\ ${ }^{1}$ Department of Physics and Astronomy and the Nebraska Center for Materials and Nanoscience, University \\ of Nebraska-Lincoln, Lincoln, Nebraska 68588-0299, USA \\ ${ }^{2}$ Department of Chemical and Biomolecular Engineering and the Nebraska Center for Materials and \\ Nanoscience, University of Nebraska-Lincoln, Lincoln, Nebraska 68588-0643, USA
}

(Received 3 May 2011; accepted 22 September 2011; published online 13 October 2011)

\begin{abstract}
We report controllable tuning of the room temperature photoluminescence band of CdSe semiconductor quantum dots embedded in thin films of ferroelectric copolymer of vinylidene fluoride and trifluoroethylene made by Langmuir-Blodgett deposition. The high breakdown strength of the polymer permits the application of electric fields of up to $400 \mathrm{MV} / \mathrm{m}$ and results in a shift in the photoluminescence peak by up to $9 \mathrm{~nm}$, nearly half the fluorescence band width. Moreover, we found that at these high electric fields, the Stark effect exhibits unusual fourth power dependence. (C) 2011 American Institute of Physics. [doi:10.1063/1.3651322]
\end{abstract}

Semiconductor nanostructures, especially nanocrystal quantum dots (QDs), are promising sources of narrowband photoluminescence due to their high quantum efficiency, stability, and spectral tunability. ${ }^{1}$ Already widely used in life sciences, ${ }^{2,3}$ quantum dots are also very promising for applications as electronic devices, ${ }^{4}$ light emitters, ${ }^{5}$ and harvesters. ${ }^{6}$ Due to quantum confinement, the optical properties of quantum dots can be tuned over a broad range by controlling size and morphology during the synthesis. ${ }^{1}$ Once synthesized, however, the optical properties of quantum dots are essentially fixed and cannot be easily tuned further. It would be technologically attractive to control the properties of quantum dots electrically and tune them on demand even inside devices. Such control is possible through the Stark effect by applying an external electric field, ${ }^{7-9}$ which causes an additional distortion of the electronic energy levels and can be observed as changes in the photoluminescence emission spectrum, particularly its wavelength, ${ }^{7}$ intensity, ${ }^{8,9}$ and lifetime. ${ }^{10}$ This may allow, for example, for precise control of the exciton oscillator strength ${ }^{11}$ and should lead to development of practical devices like color-tunable single-photon sources ${ }^{12}$ and optical filters. ${ }^{13}$ The limiting factor, however, is relatively low dielectric breakdown strength of materials surrounding quantum dots, typically in the range of 10-50 $\mathrm{MV} / \mathrm{m}$ for most matrix materials. ${ }^{13}$ Moreover, any dielectric mismatch between the quantum dots and the matrix further degrades the dielectric strength of the composite. ${ }^{14}$

Photoluminescence tuning in quantum dots by application of an external electric field has been demonstrated mainly on individual quantum dots at low temperatures, because the quantum dot emission is narrow and the spectral shifts are therefore easier to measure. Examples include colloidal quantum dots deposited between metal electrodes, ${ }^{7}$ self-assembled quantum dots epitaxially grown between electrodes ${ }^{15}$ or approached by the charged tip of a scanning tunneling microscope. ${ }^{16}$ The work by Empedocles and Bawendi $^{7}$ is particularly interesting. They observed electricfield induced shifts of the zero-phonon line by up to $19 \mathrm{~nm}$

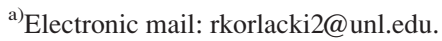

for some of the dots, the largest Stark shifts reported so far, but this was observed only for a small fraction of quantum dots studied. An interesting outcome of this work was the existence of a large heterogeneity of the Stark effects even for a rather homogenous, in terms of size and preparation procedure, sample of quantum dots. The variations in spectral response were both qualitative, with various combinations of linear and quadratic Stark effect terms, and quantitative, with very different shift values for nominally the same fields of the order of $10 \mathrm{MV} / \mathrm{m}$. This general trend was confirmed by Park et al. in room temperature studies using electric fields of up to $50 \mathrm{MV} / \mathrm{m}^{8}$ They have shown that the fieldinduced emission intensity modulations and spectral shifts were completely uncorrelated in both the magnitude and direction. Moreover, approximately half of the quantum dots responding to the external electric field with a large intensity modulation did not exhibit a measurable Stark shift.

It seems, therefore, that to tune the photoluminescence spectrum of an ensemble of quantum dots significantly, the induced electric field must be several orders of magnitude higher, which brings it well above the dielectric breakdown strength of the dielectric matrices in common use. Various solutions that have been proposed for this problem include burying quantum dots in an $\mathrm{SiO}_{2}$ matrix ${ }^{17}$ or dispersing them at the interface between two immiscible ionic liquids. ${ }^{13}$ These methods should, in principle, produce electric fields of the order of $0.1 \mathrm{GV} / \mathrm{m}$ and $1 \mathrm{GV} / \mathrm{m}$, respectively. Practical realization of the former concept did not lead, however, to an observation of the Stark shift. ${ }^{17}$ Even higher electric fields can, in principle, be achieved by embedding the quantum dots in a ferroelectric matrix, because a ferroelectric material produces a large and stable remanent polarization. In this letter, we describe a promising first step in this direction. We embedded colloidal quantum dots in a matrix consisting of ferroelectric polymers based on poly(vinylidene fluoride). ${ }^{18}$ Because the ferroelectric matrix can be permanently polarized, the field strength inside the polymer matrix can reach values well over $2 \mathrm{GV} / \mathrm{m}$, even in the absence of an external field. ${ }^{19}$ This is because ferroelectrics present a large surface charge of order $0.1 \mathrm{C} / \mathrm{m}^{2},{ }^{18}$ much larger than can typically be 
induced through an electrically biased dielectric. In addition, the dielectric mismatch between the embedded quantum dots and the matrix is reduced, because the dielectric constant of ferroelectric polymers, $\varepsilon \approx 8-10,{ }^{20}$ is comparable to the dielectric constant of typical quantum dots materials, $\varepsilon \approx 8$ (for 5-nm diameter CdSe nanocrystals). ${ }^{21}$ The uniformity of the field is an important prerequisite for achieving high fields inside a composite sample as the probability of dielectric breakdown drastically increases when highly non-uniform fields produce hot-spots with high field densities, especially prone to breakdown. ${ }^{14}$ Another advantage of ferroelectric polymers as a matrix is that they are suitable for growing high quality thin films with nanometer-level precision by LangmuirBlodgett (LB) deposition, ${ }^{20,22}$ which allows for relatively simple fabrication of composites with colloidal quantum dots.

The samples for this study were prepared using commercially available materials: CdSe nanocrystal quantum dots (5.5-6.0 nm, Ocean NanoTech, LLC, obtained as a toluene dispersion with the concentration of approximately $2.5 \mathrm{mg} /$ $\mathrm{ml}$ and used as such) and a ferroelectric copolymer of $65 \%$ vinylidene fluoride and 35\% trifluoroethylene (Kunshan Hisense Electronics Co., Ltd., Shanghai). A $0.2 \%$ solution of the polymer in dimethylsulfoxide (DMSO) has been used for LB deposition at the surface pressure of $5 \mathrm{mN} / \mathrm{m}$ (horizontal or Schaefer deposition scheme used) using an automated LB trough (NIMA 622 C) filled with purified water $(18 \mathrm{M} \Omega \cdot \mathrm{cm})$ stabilized at $25^{\circ} \mathrm{C}$. The samples were prepared on glass substrates covered with transparent and conductive layer of indium-tin oxide (ITO; $25 \mathrm{~nm}$ thick). We considered two alternative sample designs, both producing capacitors with composite dielectrics approximately $60 \mathrm{~nm}$ thick. The samples (Fig. 1(a)) consisted of either a single layer of quantum dots sandwiched between two thick layers of ferroelectric polymers LB films (herein called single-layer samples) or

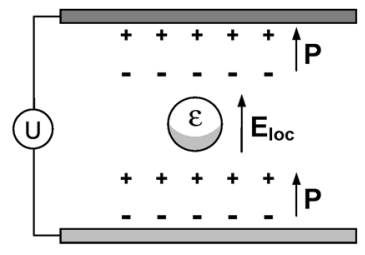

(a)

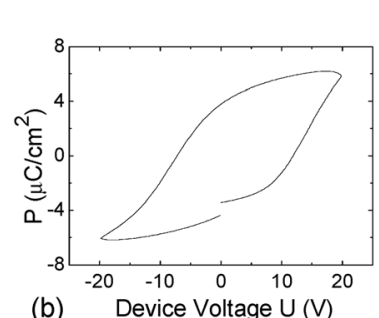

(b) Device Voltage U (V)

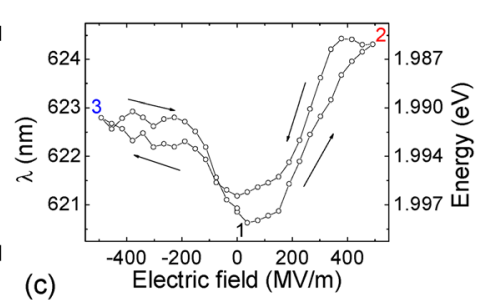

Energy (eV)

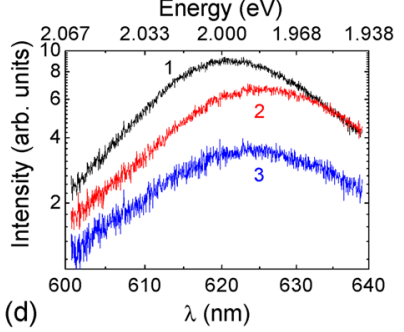

FIG. 1. (Color online) Idealized diagram of the sample structure showing a model dielectric quantum dot embedded between two slabs of polarized ferroelectric. The electrical bias $U$ is applied across the electrodes (grey). (b) Ferroelectric polarization hysteresis loop from a 60 -nm thick single-layer nanocomposite sample. (c) Shift of the peak wavelength of the photoluminescence spectrum with applied voltage and (d) photoluminescence spectra for a 60-nm thick nanocomposite sample composed of single-layer of CdSe quantum dots embedded in P(VDF-TrFE) copolymer. The sequence of voltage application, as indicated by the arrows, was from 1 to 2 to 3. 1, 2, and 3 are characteristic points on the curve in (c); for each of them, the corresponding spectra are shown in (d). multiple alternating layers of quantum dots and polymer (multi-layer samples). The typical deposition scheme to obtain a single-layer sample was 20 monolayers of ferroelectric polymer followed by spin-coating of a single layer of quantum dots at the rotational speed $1200 \mathrm{rpm}$ (the ferroelectric polymer is not soluble in toluene and therefore this step does not destroy the layer deposited previously) and another 20 monolayers of the polymer. Multi-layer samples were made by alternating deposition of 6 monolayers of polymer and a single layer of quantum dots for a total of 36 monolayers of polymer and 5 layers of quantum dots. The samples were then annealed for $1 \mathrm{~h}$ at $135^{\circ} \mathrm{C}$ and a top aluminum electrode $(50 \mathrm{~nm}$ to $100 \mathrm{~nm})$ was deposited using vacuum evaporation. The method of preparing ferroelectric polymer capacitors is described in greater detail elsewhere. ${ }^{23}$ The polarization hysteresis loops were obtained using a ferroelectric capacitor test system (Premier II, Radiant Technologies, Inc.). Photoluminescence measurements were performed using a custom-built laser confocal microscope. The excitation beam from an argon ion laser operating at a wavelength of $488 \mathrm{~nm}$ was focused on the sample with an objective lens (0.75 numerical aperture) that was also used also to collect the emission from the sample. The photoluminescence emission was filtered from the excitation light by a sharp-edge long-pass filter (RazorEdge 488, Semrock, Inc.) and recorded using a monochromator (SpectraPro SP-2500i, PI Acton) equipped with a grating with 1200 lines $/ \mathrm{mm}$ and a thermoelectrically cooled CCD detector with 1024 pixels along the dispersion axis (SpectrumOne, Jobin-Yvon, Inc.). The sample was illuminated by the excitation beam only during acquisition of the photoluminescence spectra $(2 \mathrm{~s}$ for each spectrum). The waiting time after each change of voltage was $100 \mathrm{~s}$. Results of photoluminescence measurements were plotted as a function of an average electric field obtained by dividing the actual electrical bias on the sample by the approximate sample thickness.

The representative polarization hysteresis loop (Fig. 1(b)) from one of the single-layer composite samples demonstrates that ferroelectric polarization $P$ can be readily and repeatably switched between opposing states. An electrically polarized dielectric material has surface charge density $\sigma=P$ on opposing surfaces due to the bulk alignment of electric dipoles. Therefore, if a particle with dielectric constant $\varepsilon$ is placed between two slabs of dielectric polarized in the same direction (see Fig. 1(a)), it will in effect be bounded by oppositely charged sheets that will tend to produce a large electric field of order $\sigma / \varepsilon \varepsilon_{0}$ inside the particle. We would then expect that for a single layer of quantum dots embedded in a relatively thick ferroelectric matrix, the photoluminescence spectrum from the quantum dots will exhibit hysteresis following the ferroelectric hysteresis loop due to the Stark effect. This hysteresis can indeed be seen from single-layer samples (see Fig. 1(c)).

One of the key features of multi-layer samples is the separation of the effects of external bias from the local ferroelectric polarization. The photoluminescence spectra from the QDs in these samples respond more directly to the external bias than to the polarization, as is evident by the relatively small hysteresis in the photoluminescence peak position during a voltage cycling (Fig. 2). Interestingly, the dominant component of this dependence is proportional to 

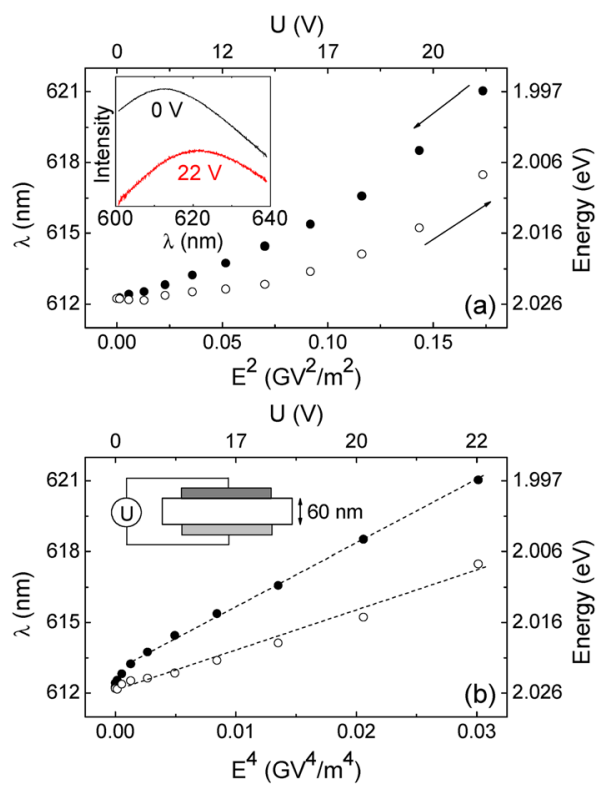

FIG. 2. (Color online) Shift of the peak wavelength of the photoluminescence spectrum with applied voltage for a 60-nm thick multi-layer nanocomposite sample with CdSe quantum dots embedded in P(VDF-TrFE) copolymer. The shift is plotted as function of second (a) and fourth (b) power of the electric field. The inset to (a) shows two representative photoluminescence spectra recorded at zero electrical bias (maximum at $612 \mathrm{~nm}$ ) and $22-\mathrm{V}$ electrical bias $(621 \mathrm{~nm})$. The measurement performed during a single continuous voltage sweep from $+22 \mathrm{~V}$ to $-22 \mathrm{~V}$, as shown by arrows in (a). The data points for positive and negative bias do not overlap mainly due to a gradual degradation of the sample during the measurement (see Fig. 3).

the fourth power of the external field, while all previous studies have shown that for quantum dots exposed to external fields, this dependence could be a various combination of linear and quadratic Stark effect. Very clear character of the fourth-power dependence suggests that we observe a nonlinear interaction between the polymer and the embedded quantum dots rather than result of a simple distortion of the polymer layers by extremely high fields.

The shift of the photoluminescence peak is accompanied by a decrease in its intensity (quenching) and slow degradation of the quantum dots on cycling the voltage, both changes evident in Fig. 3. Quenching of the photoluminescence upon application of an external field is a common phenomenon. Electrons and holes have opposite charges and therefore an external electric field tends to separate them, reducing the overlap between their wave functions and lowering probability of radiative recombination. The degradation of quantum dots in our experiments, visible particularly as a gradual decay of photoluminescence intensity in Fig. 3, is caused by the fact that for the sake of conceptual simplicity, we did not use quantum dots that are protected by thick capping layers of another higher bandgap semiconductor material (usually zinc sulphide, $\mathrm{ZnS}$, in case of $\mathrm{CdSe}$ ). Such a protection remarkably improves the quantum confinement and photochemical stability of the quantum dot cores, for example, by sealing surface defects and preventing reactions with oxygen present in their local environment, but would certainly interfere with the distribution of electric field inside the composite matrix. Therefore, we used CdSe nanocrystals that were coated with organic ligands (octadecylamine) to improve solubility and prevent agglomeration.

In summary, we have observed pronounced Stark shifts of the photoluminescence from quantum dots embedded in a ferro-

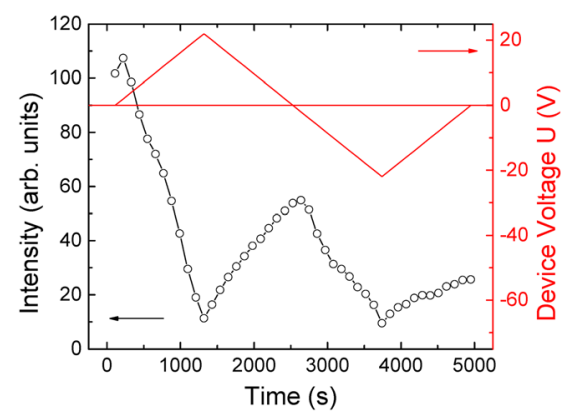

FIG. 3. (Color online) Integrated intensity of the photoluminescence spectrum (left-hand scale) as the applied voltage (right-hand scale) was slowly cycled for a $60-\mathrm{nm}$ thick nanocomposite sample composed of with CdSe quantum dots embedded in P(VDF-TrFE) copolymer. The data points in Fig. 2 were obtained from spectra recorded for the middle part of the cycle, from 1300 to 3700 on the time scale.

electric polymer matrix. Our results are remarkable both quantitatively, because the maximum shift was $9 \mathrm{~nm}$, nearly half the fluorescence band width of $20 \mathrm{~nm}$, and qualitatively, because the dominant fourth-order Stark effect was not previously observed, nor expected. This is a promising starting point, which may result in numerous applications of this rarely considered combination of modern materials, ferroelectrics and quantum dots, in tunable optical filters and light emitting devices.

The work was supported by the Nebraska Research Initiative. The authors thank Radiant Technologies, Inc. for making their ferroelectric tester accessible for measurements.

${ }^{1}$ A. P. Alivisatos, Science 271, 933 (1996).

${ }^{2}$ M. Bruchez, Jr., M. Moronne, P. Gin, S. Weiss, and A. P. Alivisatos, Science 281, 2013 (1998).

${ }^{3}$ X. Michalet, F. F. Pinaud, L. A. Bentolila, J. M. Tsay, S. Doose, J. J. Li, G. Sundaresan, A. M. Wu, S. S. Gambhir, and S. Weiss, Science 307, 538 (2005).

${ }^{4}$ T. Fujisawa, T. Hayashi, R. Tomita, and Y. Hirayama, Science 312, 1634 (2006).

${ }^{5}$ S. Coe, W.-K. Woo, M. Bawendi, and V. Bulović, Nature (London) 420, 800 (2002).

${ }^{6}$ I. Gur, N. A. Fromer, M. L. Geier, and A. P. Alivisatos, Science 310, 462 (2005).

${ }^{7}$ S. A. Empedocles and M. G. Bawendi, Science 278, 2114 (1997).

${ }^{8}$ S.-J. Park, S. Link, W. L. Miller, A. Gesquiere, and P. F. Barbara, Chem. Phys. 341, 169 (2007).

${ }^{9}$ H. Huang, A. Dorn, G. P. Nair, V. Bulović, and M. G. Bawendi, Nano Lett. 7, 3781 (2007).

${ }^{10}$ Y. Ohara, T. Nakabayashi, K. Iwasaki, T. Torimoto, B. Ohtani, T. Hiratani, K. Konishi, and N. Ohta, J. Phys. Chem. B 110, 20927 (2006).

${ }^{11}$ A. F. Jarjour, R. A. Oliver, A. Tahraoui, M. J. Kappers, C. J. Humphreys, and R. A. Taylor, Phys. Rev. Lett. 99, 197403 (2007).

${ }^{12}$ M. J. Fernée, H. Rubinsztein-Dunlop, and G. J. Milburn, Phys. Rev. A 75, 43815 (2007).

${ }^{13}$ M. E. Flatté, A. A. Kornyshev, and M. Urbakh, Proc. Natl. Acad. Sci. U.S.A. 105, 18212 (2008).

${ }^{14}$ J. Y. Li, L. Zhang, and S. Ducharme, Appl. Phys. Lett. 90, 132901 (2007).

${ }^{15}$ J. Seufert, M. Obert, M. Scheibner, N. A. Gippius, G. Bacher, A. Forchel, T. Passow, K. Leonardi, and D. Hommel, Appl. Phys. Lett. 79, 1033 (2001).

${ }^{16}$ U. Håkanson, H. Håkanson, M. K.-J. Johansson, L. Samuelson, and M.-E. Pistol, J. Vac. Sci. Technol. B 21, 2344 (2003).

${ }^{17}$ A. W. Achtstein, H. Karl, and B. Stritzker, Appl. Phys. Lett. 89, 61103 (2006).

${ }^{18}$ A. J. Lovinger, Science 220, 1115 (1983).

${ }^{19}$ L. M. Blinov, K. A. Verkhovskaya, S. P. Palto, and A. V. Sorokin, Appl. Phys. Lett. 68, 2369 (1996).

${ }^{20}$ A. V. Bune, V. M. Fridkin, S. Ducharme, L. M. Blinov, S. P. Palto, A. V. Sorokin, S. G. Yudin, and A. Zlatkin, Nature (London) 391, 874 (1998).

${ }^{21}$ T. D. Krauss and L. E. Brus, Phys. Rev. Lett. 83, 4840 (1999); T. D. Krauss and L. E. Brus, ibid. 84, 1638(E) (2000).

${ }^{22}$ S. Palto, L. Blinov, A. Bune, E. Dubovik, V. Fridkin, N. Petukhova, K. Verkhovskaya, and S. Yudin, Ferroelectr., Lett. Sect. 19, 65 (1995).

${ }^{23}$ L. M. Blinov, V. M. Fridkin, S. P. Palto, A. V. Bune, P. A. Dowben, and S. Ducharme, Phys. Usp. 43, 243 (2000). 